\title{
Size-dependent thermoelasticity
}

\section{Abstract}

In this paper a consistent theory is developed for size-dependent thermoelasticity in heterogeneous anisotropic solids. This theory shows that the temperature change can create not only thermal strains, but also thermal mean curvatures in the solids. This formulation is based on the consistent size-dependent continuum mechanics in which the couple-stress tensor is skew-symmetric. Here by including scale-dependent measures in the energy and entropy equations, the general expressions for force- and couplestresses, as well as entropy density, are obtained. Next, for the linear material the constitutive relations and governing coupled size-dependent thermoelasticity equations are developed. For linear material, one can see that the thermal properties are characterized by the classical symmetric thermal expansion tensor and the new size-dependent skew-symmetric thermal flexion tensor. Thus, for the most general anisotropic case, there are nine independent thermoelastic constants. Interestingly, for isotropic and cubic materials the thermal flexion tensor vanishes, which shows there is no thermal mean curvature.

Keywords: scaling, size effect, thermoelasticity

\section{Ali R. Hadjesfandiari ${ }^{*}$}

Department of Mechanical and Aerospace Engineering

University at Buffalo, State University of New York

Buffalo, NY 14260 USA

Author e-mail: ah@buffalo.edu

\section{INTRODUCTION}

Within the framework of the consistent continuum mechanics, couple-stresses inevitably appear along with the force-stresses to describe the interaction of constituents of the matter. Accordingly, the force-stress tensor is not symmetric. Neglecting the couple-stresses results in what is called the classical continuum mechanics, which provides a reasonable basis for analyzing the behavior of materials when the size-dependency can be ignored. However, experiments usually do not agree with the classical theory in the regions, where the gradients of strains and rotations are very high. These regions include areas near the boundary surfaces, such as holes, notches and cracks, where the size dependency is observed. This may be especially important in fracture mechanics, where considering the couple stresses not only accounts for the size dependency, it also provides new failure criteria and crack growth law. Therefore, a consistent size-dependent continuum theory is necessary to account for the length scale effect due to the microstructure of materials. The new progress in micromechanics, nanomechanics and nanotechnology requires this advanced size dependent modeling of continuum in different branches of multi-physics disciplines, such as thermoelasticity. 
Thermoelasticity is a multi-physics discipline, which investigates the interaction of the field of deformation with the field of temperature. It combines, on the basis of the thermodynamics of the irreversible processes, two separately physical phenomena, namely the theory of elasticity and the theory of heat conduction. Classical thermoelasticity describes the relation between temperature change and strain in solids (Biot, 1956; Nowacki, 1962). However, the classical theory cannot address the size dependency, because it does not include any length dependent parameter in its formulation. Therefore, it is necessary to develop a sizedependent thermoelasticity, which accounts for the microstructure of the material by introducing higher gradients of deformation. It is interesting to note that the classical theory of thermoelasticity predicts that for two-dimensional simply connected isotropic regions, a steady state temperature field with zero boundary tractions will not affect the in-plane stress field. This rather surprising conclusion is the result of simplicity of classical thermoelasticity. The new size-dependent thermoelasticity predicts a stress boundary layer for this case, which is more important in small scale bodies.

Ahmadi and Firoozbakhsh (1975) have developed a size-dependent thermoelasticity theory by considering the first gradient of the strain tensor. This development is within the framework of the strain gradient theory (Mindlin, 1964; Mindlin and Eshel, 1968). However, the strain gradient tensor is not the fundamental measure of deformation in a consistent sizedependent continuum theory. The micropolar theory has also been extended to include thermal effects by Nowacki (1966a,b,c) and Eringen (1970). Nevertheless, microrotation, which brings extraneous degrees of freedom, is not a proper continuum mechanical concept.

Recently, Hadjesfandiari and Dargush (2011) have developed the consistent continuum mechanics for solids. In this theory, the couple-stress tensor is skew-symmetric and the body couple is not distinguishable from the body force. The work also demonstrates that the stresses are fully determinate, and the measure of deformation energetically-conjugate to couple-stress is the skew-symmetrical mean curvature tensor. This theory involves only true continuum kinematical quantities without recourse to any additional artificial degrees of freedom. An initial incomplete version of this theory was developed by Mindlin and Tiersten (1962), and Koiter (1964), which used the displacement field, its corresponding true continuum rotation and the gradient of the rotation as the curvature tensor. However, this curvature tensor is not a proper measure of deformation and creates indeterminacy in the couplestress tensor.

Establishing the skew-symmetric character of the couple-stress tensor resolves the quest for the consistent size-dependent continuum mechanics, which provides a fundamental basis for the development of size-dependent material response. Therefore, this development can be extended quite naturally into many branches of continuum mechanics involving different multi-physics disciplines. Hadjesfandiari (2013) has already developed a size-dependent piezoelectricity for dielectric materials. Here we concentrate to develop the coupled sizedependent thermoelasticity for solids.

Latin American Journal of Solids and Structures 11 (2014) 1679-1708 
In the following section, we provide an overview of the thermomechanical equations. This includes the equations for the kinematics, mechanics and thermodynamics of size-dependent small deformation continuum mechanics. Here by including scale-dependent measures in the energy and entropy equations, the general thermoelastic expressions for force- and couplestresses, as well as entropy density, are obtained. In Section 3, the constitutive relations for linear size-dependent heterogeneous anisotropic thermoelastic materials also are derived along with the governing equations. Section 4 provides the general theory of size-dependent thermoelasticity for linear isotropic material. This section includes the size-dependent thermoelastic waves as well. Finally, Section 5 contains a summary and some general conclusions.

\section{GOVERNING SIZE-DEPENDENT THERMOMECHANICAL EQUATIONS}

Consider a heterogeneous anisotropic elastic solid material occupying a volume $V$ bounded by a surface $A$. Assume the body is undeformed and is stress-free at a uniform absolute temperature $T_{0}$ when there is no external force. This initial state is referred as the reference or natural state of the body, and the absolute temperature $T_{0}$ is called the reference temperature. When the body is subjected to external forces and heat sources, it undergoes a temperature change field $\vartheta=T-T_{0}$, and an accompanying deformation specified by the displacement field $\mathbf{u}$. These quantities may depend on position and time. Here, we assume infinitesimal deformation and temperature changes, where

$$
\left|\frac{\partial u_{i}}{\partial x_{j}}\right| \ll<1,\left|\frac{\partial^{2} u_{i}}{\partial x_{j} \partial x_{k}}\right| \ll<\frac{1}{l_{S}},\left|\frac{\vartheta}{T_{0}}\right|=\left|\frac{T-T_{0}}{T_{0}}\right| \ll<1
$$

The parameter $l_{S}$ represents the smallest characteristic length in the body. By these assumptions, the mechanical and thermal properties of the material can be taken constants during deformation and temperature change.

The infinitesimal strain and rotation tensors are defined as

$$
\begin{aligned}
& e_{i j}=u_{(i, j)}=\frac{1}{2}\left(u_{i, j}+u_{j, i}\right) \\
& \omega_{i j}=u_{[i, j]}=\frac{1}{2}\left(u_{i, j}-u_{j, i}\right)
\end{aligned}
$$

respectively. Since the true tensor $\omega_{i j}$ is skew-symmetrical, one can introduce its corresponding dual pseudo rotation vector as

$$
\omega_{i}=\frac{1}{2} \varepsilon_{i j k} \omega_{k j}
$$


where $\varepsilon_{i j k}$ is the permutation tensor or Levi-Civita symbol. The consistent infinitesimal pseudo skew-symmetric mean curvature tensor defined by Hadjesfandiari and Dargush (2011) as

$$
\kappa_{i j}=\omega_{[i, j]}=\frac{1}{2}\left(\omega_{i, j}-\omega_{j, i}\right)
$$

Since this tensor is also skew-symmetrical, its corresponding dual true mean curvature vector is

$$
\kappa_{i}=\frac{1}{2} \varepsilon_{i j k} \kappa_{k j}
$$

By some manipulation, one can obtain

$$
\kappa_{i}=\frac{1}{2} \omega_{j i, j}=\frac{1}{4} u_{j, j i}-\frac{1}{4} \nabla^{2} u_{i}
$$

The external forces, heat sources and heating produce internal stresses and heat fluxes in the body. The internal stresses are represented by true force-stress $\sigma_{i j}$ and pseudo couplestress $\mu_{i j}$ tensors. The components of these force-stress and couple-stress tensors are shown in Fig. 1. The force-traction vector $t_{i}^{(n)}$ and moment-traction vector $m_{i}^{(n)}$ through a surface element $d A$ in the volume with outward directed unit normal vector $n_{i}$ are given 

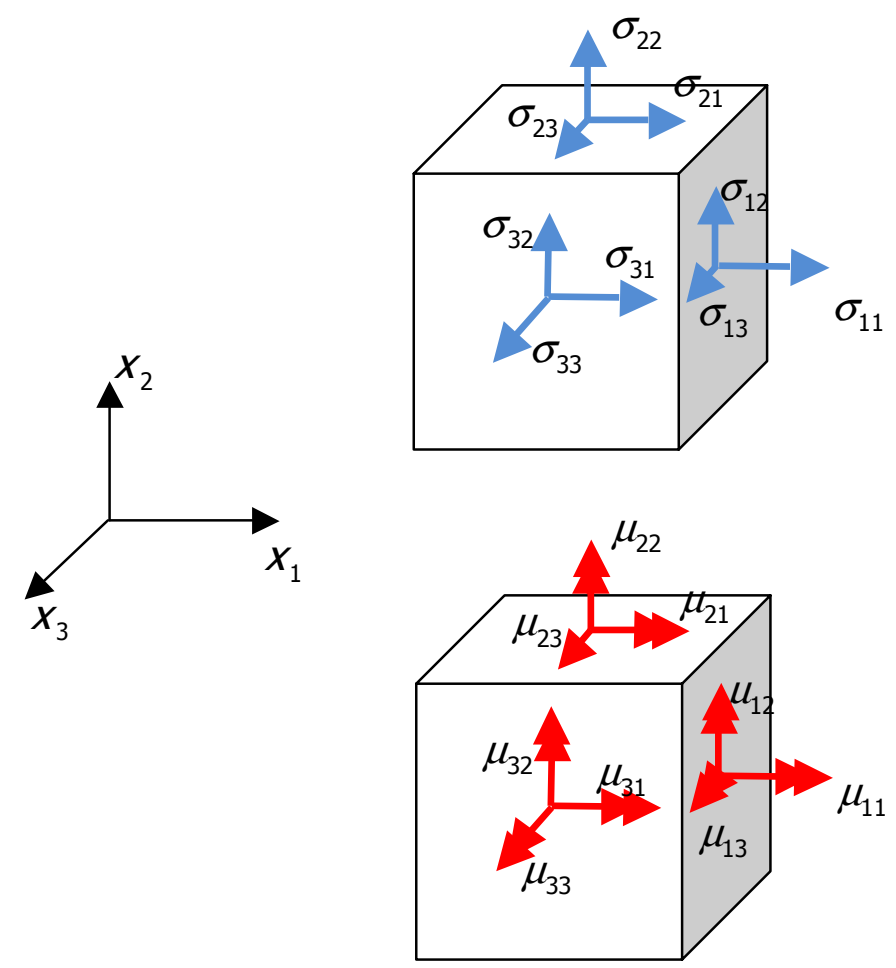

Figure 1 Components of force- and couple-stress tensors in the original inconsistent theory.

$$
\begin{gathered}
t_{i}^{(n)}=\sigma_{j i} n_{j} \\
m_{i}^{(n)}=\mu_{j i} n_{j}
\end{gathered}
$$

The force-stress tensor $\sigma_{j i}$ is generally non-symmetric and can be decomposed as

$$
\sigma_{j i}=\sigma_{(j i)}+\sigma_{[j i]}
$$

where $\sigma_{(j i)}$ and $\sigma_{[j i]}$ are the symmetric and skew-symmetric parts, respectively. Based on the new development in size-dependent continuum mechanics (Hadjesfandiari and Dargush, 2011), the pseudo couple-stress tensor $\mu_{i j}$ is skew-symmetrical

$$
\mu_{j i}=-\mu_{i j}
$$

The components of the force-stress $\sigma_{i j}$ and couple-stress $\mu_{i j}$ tensors in this consistent theory are shown in Fig. 2. 


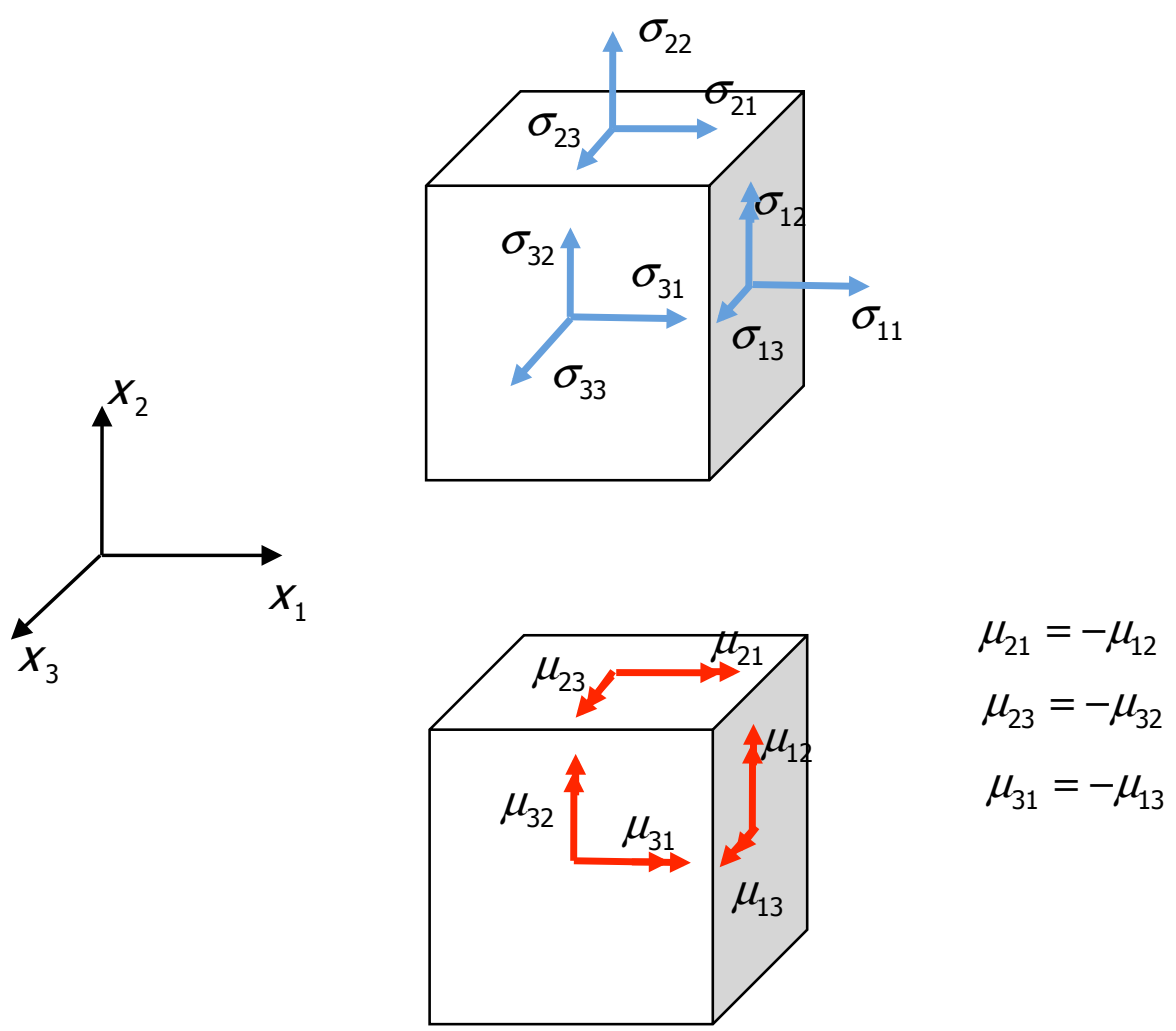

Figure 2 Components of force- and couple-stress tensors in the present consistent theory.

Since $\mu_{i j}$ is skew-symmetric, the moment-traction $m_{i}^{(n)}$ given by (9) is tangent to the surface. As a result, the couple-stress tensor $\mu_{i j}$ creates only bending moment-traction on any arbitrary surface in matter. The force-traction $t_{i}^{(n)}$ and the consistent bending momenttraction $m_{i}^{(n)}$ acting on an arbitrary surface with unit normal vector $n_{i}$ are shown in Fig. 3.

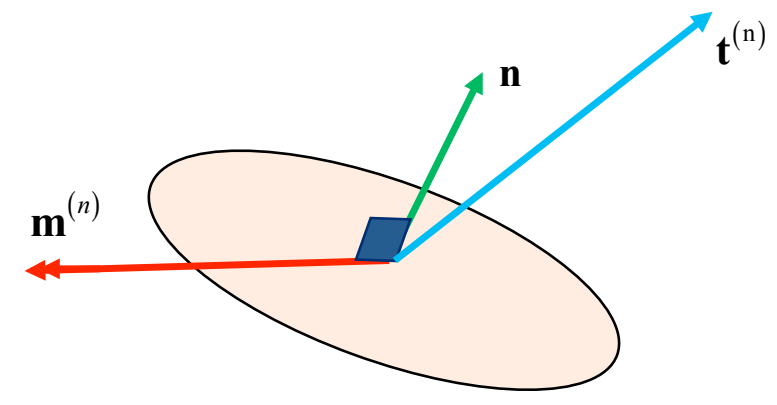

Figure 3 Force-traction $\mathbf{t}^{(n)}$ and the consistent bending moment-traction $\mathbf{m}^{(n)}$. 
The true couple-stress vector $\mu_{i}$ dual to the pseudo-tensor $\mu_{i j}$ is defined as

$$
\mu_{i}=\frac{1}{2} \varepsilon_{i j k} \mu_{k j}
$$

Consequently, the surface moment-traction vector tangent to the surface $m_{i}^{(n)}$ reduces to

$$
m_{i}^{(n)}=\mu_{j i} n_{j}=\varepsilon_{i j k} n_{j} \mu_{k}
$$

To formulate the fundamental equations, we consider an arbitrary part of this heterogeneous anisotropic elastic body occupying a volume $V_{a}$ enclosed by boundary surface $A_{a}$. In infinitesimal deformation theory, the displacement vector field $\mathbf{u}$ is so small that the velocity and acceleration fields can be approximated by $\dot{\mathbf{u}}$ and $\ddot{\mathbf{u}}$, respectively. As a result, the linear and angular equations of motion for this part of the body are written as

$$
\begin{gathered}
\int_{A_{a}} t_{i}^{(n)} d A+\int_{V_{a}} \rho f_{i} d V=\int_{V_{a}} \rho \ddot{u}_{i} d V \\
\int_{A_{a}}\left[\varepsilon_{i j k} x_{j} t_{k}^{(n)}+m_{i}^{(n)}\right] d A+\int_{V_{a}} \varepsilon_{i j k} x_{j} \rho f_{k} d V=\int_{V_{a}} \varepsilon_{i j k} x_{j} \rho \ddot{u}_{k} d V
\end{gathered}
$$

where $f_{i}$ is the body force per unit mass of the body, and $\rho$ is the mass density. Hadjesfandiari and Dargush (2011) have shown that the body couple density is not distinguishable from body force in size-dependent couple stress continuum mechanics and its effect is simply equivalent to a system of body force and surface traction.

By using Eqs. (8) and (9) for tractions in the equations of motion (14) and (15), along with the divergence theorem, and noticing the arbitrariness of volume $V_{a}$, we finally obtain the differential form of the equations of motion as

$$
\begin{aligned}
& \sigma_{j i, j}+\rho f_{i}=\rho \ddot{u}_{i} \\
& \mu_{j i, j}+\varepsilon_{i j k} \sigma_{j k}=0
\end{aligned}
$$

We note that the angular equation of motion (17) gives the skew-symmetric part of the force-stress tensor as

$$
\sigma_{[j i]}=-\frac{1}{2} \varepsilon_{i p q} \mu_{q p, j}=-\mu_{[i, j]}
$$


Therefore, the independent linear equation of motion reduces to

$$
\left[\sigma_{(j i)}+\mu_{[j, i]}\right]_{, j}+\rho f_{i}=\rho \ddot{u}_{i}
$$

Now, we consider the first law of thermodynamics for this body. Heat conduction arises from the irreversible transfer of heat energy due to the temperature gradient in the body. The heat conduction energy flow is represented by heat flux or thermal flux vector $q_{i}$. The amount of heat flux energy flow $q^{(n)}$ through the surface element $d A$ with outward directed unit normal vector $n_{i}$ is given by (Carslaw and Jaeger, 1959)

$$
q^{(n)}=q_{i} n_{i}
$$

The energy equation for the arbitrary volume $V_{a}$ can be written as

$$
\frac{\partial}{\partial t} \int_{V_{a}}\left(\frac{1}{2} \rho \dot{u}_{i} \dot{u}_{i}+U\right) d V=\int_{A_{a}}\left(t_{i}^{(n)} \dot{u}_{i}+m_{i}^{(n)} \dot{\omega}_{i}-q^{(n)}\right) d A+\int_{V_{a}}\left(\rho f_{i} \dot{u}_{i}+Q\right) d V
$$

where $U$ is the internal energy per unit volume and $Q$ is the quantity of heat generated in unit time and unit volume. This equation shows that the rate of change of total energy of the system in the volume $V_{a}$ is equivalent to the power of the external forces, moments and the heat power received by thermal conduction. By substituting from Eqs. (8), (9) and (20), this equation becomes

$$
\frac{\partial}{\partial t} \int_{V_{a}}\left(\frac{1}{2} \rho \dot{u}_{i} \dot{u}_{i}+U\right) d V=\int_{A_{a}}\left(\sigma_{j i} \dot{u}_{i} n_{j}+\mu_{j i} \dot{\omega}_{i} n_{j}-q_{i} n_{i}\right) d A+\int_{V_{a}}\left(\rho f_{i} \dot{u}_{i}+Q\right) d V
$$

Then by using the divergence theorem and equations of motion (16) and (17), and noticing the arbitrariness of volume $V_{a}$, one can obtain the energy balance in differential form

$$
\dot{U}=\sigma_{(j i)} \dot{e}_{i j}+\mu_{j i} \dot{\kappa}_{i j}-q_{i, i}+Q
$$

The second law of thermodynamics for this volume can be written as

$$
\frac{\partial}{\partial t} \int_{V_{a}} S d V \geq \int_{A_{a}} \frac{-q^{(n)}}{T} d A+\int_{V_{a}} \frac{Q}{T} d V
$$


where $S$ is the internal entropy per unit volume. By using Eq. (20) for the heat flux $q^{(n)}$ in Eq. (24), along with the divergence theorem, and noticing the arbitrariness of volume $V_{a}$, we obtain the Clausius-Duhem inequality

$$
\dot{S} \geq-\left(\frac{q_{i}}{T}\right)_{, i}+\frac{Q}{T}
$$

which can also be written as

$$
\dot{S} \geq-\frac{q_{i, i}}{T}+\frac{q_{i} T_{, i}}{T^{2}}+\frac{Q}{T}
$$

By combining Eqs. (23), and (26) to eliminate $Q$, we have

$$
-(\dot{U}-T \dot{S})+\sigma_{(j i)} \dot{e}_{i j}+\mu_{j i} \dot{\kappa}_{i j}-\frac{q_{i} T_{, i}}{T} \geq 0
$$

It turns out that for our development we should define the Helmholtz free energy density $F$ by using the Legendre transformation as

$$
F=U-T S
$$

By differentiating with respect to time, we obtain

$$
\dot{F}=\dot{U}-S \dot{T}-\dot{S} T
$$

Then by introducing Eq. (29) in Eq. (27), we obtain

$$
-(\dot{F}+S \dot{T})+\sigma_{(j i)} \dot{e}_{i j}+\mu_{j i} \dot{\kappa}_{i j}-\frac{q_{i} T_{, i}}{T} \geq 0
$$

However, for the simple elastic body the free energy density is a function of the independent variables $e_{i j}, \kappa_{i j}$ and $T$; that is

$$
F=F\left(e_{i j}, \kappa_{i j}, T\right)
$$

If we differentiate $F$ with respect to time, we obtain 


$$
\mathscr{R}=\left(\frac{\partial F}{\partial e_{i j}}\right)_{\kappa_{i j}, T} \underset{i j}{\&}+\left(\frac{\partial F}{\partial \kappa_{i j}}\right)_{e_{i j}, T} \underset{i j}{\&}+\left(\frac{\partial F}{\partial T}\right)_{e_{i j}, \kappa_{i j}} \not \&
$$

This can be simply written as

$$
\dot{F}=\frac{\partial F}{\partial e_{i j}} \dot{e}_{i j}+\frac{\partial F}{\partial \kappa_{i j}} \dot{\kappa}_{i j}+\frac{\partial F}{\partial T} \dot{T}
$$

Then by using Eq. (33) in Eq. (30), we obtain

$$
\left(\sigma_{(j i)}-\frac{\partial F}{\partial e_{i j}}\right) \dot{e}_{i j}+\left(\mu_{j i}-\frac{\partial F}{\partial \kappa_{i j}}\right) \dot{\kappa}_{i j}-\left(S+\frac{\partial F}{\partial T}\right) \dot{T}-\frac{q_{i} T_{, i}}{T} \geq 0
$$

This inequality should be satisfied for all independent arbitrary rates $\dot{e}_{i j}, \dot{\kappa}_{i j}$ and $\dot{T}$. Hence, their corresponding coefficients in Eq. (34) must vanish. Therefore, we find the following general constitutive relations for the symmetric part of the force-stress tensor $\sigma_{(j i)}$, couple-stress tensor $\mu_{j i}$ and entropy $S$ :

$$
\begin{gathered}
\sigma_{(j i)}=\frac{1}{2}\left(\frac{\partial F}{\partial e_{i j}}+\frac{\partial F}{\partial e_{j i}}\right) \\
\mu_{j i}=\frac{1}{2}\left(\frac{\partial F}{\partial \kappa_{i j}}-\frac{\partial F}{\partial \kappa_{j i}}\right) \\
S=-\frac{\partial F}{\partial T}
\end{gathered}
$$

As a result, the Clausius-Duhem inequality Eq. (34) reduces to

$$
q_{i} T_{, i} \leq 0
$$

which means the transfer of heat in the opposite direction of temperature gradient. This is the simple result of the second law of thermodynamics. 
The relations (28) and (31) show that

$$
U=U\left(e_{i j}, \kappa_{i j}, S\right)
$$

As a result, we have the rate equation

$$
\dot{U}=\sigma_{(j i)} \dot{e}_{i j}+\mu_{j i} \dot{\kappa}_{i j}+T \dot{S}
$$

By using Eq. (40) in the energy balance Eq. (23), we obtain the equation of entropy balance in differential form

$$
T \dot{S}=-q_{i, i}+Q
$$

Let us return to the constitutive equations (35-37). If we further agree to construct the functional $F$, such that

$$
\begin{gathered}
\frac{\partial F}{\partial e_{i j}}=\frac{\partial F}{\partial e_{j i}} \\
\frac{\partial F}{\partial \kappa_{i j}}=-\frac{\partial F}{\partial \kappa_{j i}}
\end{gathered}
$$

we can write in place of Eqs. (35) and (36)

$$
\begin{gathered}
\sigma_{(j i)}=\frac{\partial F}{\partial e_{i j}} \\
\mu_{j i}=\frac{\partial F}{\partial \kappa_{i j}}
\end{gathered}
$$

It should be noticed that if we consider the Helmholtz free energy in the form $F=F\left(e_{i j}, \kappa_{i}, T\right)$, we obtain

$$
\mu_{i}=-\frac{1}{2} \frac{\partial F}{\partial \kappa_{i}}
$$


for the couple-stress vector. Then by using this in Eq. (18) we obtain the skew-symmetric part of the force-stress tensor $\sigma_{[j i]}$ as

$$
\sigma_{[j i]}=-\mu_{[i, j]}=\frac{1}{4}\left(\frac{\partial F}{\partial \kappa_{i}}\right)_{, j}-\frac{1}{4}\left(\frac{\partial F}{\partial \kappa_{j}}\right)_{, i}
$$

Therefore, for the total force-stresses, we generally have

$$
\sigma_{j i}=\frac{1}{2}\left(\frac{\partial F}{\partial e_{i j}}+\frac{\partial F}{\partial e_{j i}}\right)+\frac{1}{4}\left(\frac{\partial F}{\partial \kappa_{i}}\right)_{, j}-\frac{1}{4}\left(\frac{\partial F}{\partial \kappa_{j}}\right)_{, i}
$$

Now, we consider the entropy density function $S$. The relation (37) shows that

$$
S=S\left(e_{i j}, \kappa_{i j}, T\right)
$$

By differentiating with respect to time, we have

$$
\dot{S}=\left(\frac{\partial S}{\partial e_{i j}}\right)_{\kappa_{i j}, T} \dot{e}_{i j}+\left(\frac{\partial S}{\partial \kappa_{i j}}\right)_{e_{i j}, T} \dot{\kappa}_{i j}+\left(\frac{\partial S}{\partial T}\right)_{e_{i j}, k_{i j}} \dot{T}
$$

Using Eq. (50) in Eq. (40) for $\dot{U}$, we obtain

$$
\dot{U}=\left[T\left(\frac{\partial S}{\partial e_{i j}}\right)_{\kappa_{i j}, T}+\sigma_{(j i)}\right] \dot{e}_{i j}+\left[T\left(\frac{\partial S}{\partial \kappa_{i j}}\right)_{e_{i j}, T}+\mu_{j i}\right] \dot{\kappa}_{i j}+T\left(\frac{\partial S}{\partial T}\right)_{e_{i j}, \kappa_{i j}} \dot{T}
$$

This expression shows that the quantity $T\left(\frac{\partial S}{\partial T}\right)_{e_{i j}, x_{i j}}$ is the measure of internal energy change rate in unit volume of the body during a change in temperature at constant strain and curvature. Consequently, we realize that this quantity is the specific heat at constant strain and curvature and denote it by $c_{v}$, that is

$$
c_{v}=\left(\frac{\partial U}{\partial T}\right)_{e_{i j}, k_{i j}}=T\left(\frac{\partial S}{\partial T}\right)_{e_{i j}, \kappa_{i j}}=-T\left(\frac{\partial^{2} F}{\partial T^{2}}\right)_{e_{i j} x_{j}}
$$


Therefore, the relation for energy and entropy rate densities become

$$
\begin{gathered}
\dot{U}=\left[T\left(\frac{\partial S}{\partial e_{i j}}\right)_{\kappa_{i j}, T}+\sigma_{(j i)}\right] \dot{e}_{i j}+\left[T\left(\frac{\partial S}{\partial \kappa_{i j}}\right)_{e_{i j}, T}+\mu_{j i}\right] \dot{\kappa}_{i j}+c_{v} \dot{T} \\
\dot{S}=\left(\frac{\partial S}{\partial e_{i j}}\right)_{\kappa_{i j}, T} \dot{e}_{i j}+\left(\frac{\partial S}{\partial \kappa_{i j}}\right)_{e_{i j}, T} \dot{\kappa}_{i j}+\frac{c_{v}}{T} \dot{T}
\end{gathered}
$$

The relation (54) will be used in our development in the next section.

What has been presented so far is the size-dependent continuum mechanics theory of infinitesimal thermoelastic materials. The fundamental thermoelastic equations in the volume $V$ are

$$
\begin{gathered}
{\left[\sigma_{(j i)}+\mu_{[j, i]}\right]_{, j}+\rho f_{i}=\rho \ddot{u}_{i} \quad \text { Linear momentum balance }} \\
\dot{U}=\sigma_{(j i)} \dot{e}_{i j}+\mu_{j i} \dot{\kappa}_{i j}-q_{i, i}+Q \text { Energy balance } \\
T \dot{S}=-q_{i, i}+Q \text { Entropy balance } \\
q_{i} T_{, i} \leq 0 \text { Clausius-Duhem inequality }
\end{gathered}
$$

with the constitutive relations

$$
\begin{gathered}
\sigma_{j i}=\frac{1}{2}\left(\frac{\partial F}{\partial e_{i j}}+\frac{\partial F}{\partial e_{j i}}\right)+\frac{1}{4}\left(\frac{\partial F}{\partial \kappa_{i}}\right)_{, j}-\frac{1}{4}\left(\frac{\partial F}{\partial \kappa_{j}}\right)_{, i} \\
\mu_{i}=-\frac{1}{2} \frac{\partial F}{\partial \kappa_{i}} \\
S=-\frac{\partial F}{\partial T}
\end{gathered}
$$

We notice that the these governing equations are subject to some prescribed compatible boundary conditions on the boundary $A$. From a mathematical point of view, we can speci- 
fy either the displacement vector $u_{i}$ or the force-traction vector $t_{i}^{(n)}$, the tangential component of the rotation vector $\omega_{i}$ or the tangent moment-traction vector $m_{i}^{(n)}$, and the absolute temperature $T$ or the normal heat flux $q^{(n)}$. In a time dependent problem, we are also required to specify the initial conditions at an initial time, say $t=0$, everywhere in the volume $V$ to have a consistent initial boundary value problem. These include initial deformation $u_{i}(\mathbf{x}, 0)$, velocity $\dot{u}_{i}(\mathbf{x}, 0)$ and absolute temperature $T(\mathbf{x}, 0)$.

It is obvious that the reference or natural state is different from the initial state. The body in its natural state is without any stresses, strains and curvatures, that is

$$
\text { at } T=T_{0}, \quad e_{i j}=0, \quad \kappa_{i j}=0, \quad \sigma_{i j}=0, \quad \mu_{i j}=0 \quad \text { in } V
$$

\section{LINEAR THERMOELASTICITY THEORY}

For linear material, we consider the classical Duhamel's generalization of Fourier heat conduction law (Duhamel, 1832) as

$$
q_{i}=-k_{i j} T_{, j}
$$

where $k_{i j}$ is the thermal conductivity tensor. Therefore, the Clausius-Duhem inequality (58) reduces to

$$
k_{i j} T_{, i} T_{, j} \geq 0
$$

Based on the non-equilibrium statistical mechanics, Onsager (1931) has shown that the tensor $k_{i j}$ is symmetric

$$
k_{i j}=k_{j i}
$$

Consequently, Eq. (64) proves that the non-singular symmetric conductivity tensor $k_{i j}$ is positive definite. By introducing Eq. (63) in the entropy balance Eq. (57), we obtain

$$
T \dot{S}=\left(k_{i j} T_{, i}\right)_{, j}+Q
$$

for heterogeneous linear materials.

It should be noticed that the classical theory of generalized Fourier heat conduction (63) predicts infinite speed of heat transport, which contradicts the physical facts. As a result, 
non-classical theories have been developed to incorporate a flux rate term into the Fourier's law of heat conduction and formulated a generalized theory admitting finite speed for thermal signals (e.g., Lord and Shulman, 1967). These theories consider heat propagation as a wave phenomenon rather than a diffusion phenomenon. However, in this paper we have not included this formulation in our development. In a future work, the formulation to incorporate a flux rate term into the Fourier's law of heat conduction in the framework of size-dependent thermoeleasticity will be developed to admit finite speed for thermal signals.

Now let us expand the Helmholtz free energy $F=F\left(e_{i j}, \kappa_{i j}, T\right)$ into the Taylor expansion in the neighborhood of the reference state, where $\left(e_{i j}=0, \kappa_{i j}=0, T=T_{0}\right)$, that is

$$
\begin{aligned}
& F\left(e_{i j}, \kappa_{i j}, T\right)=F\left(0,0, T_{0}\right)+\frac{\partial F\left(0,0, T_{0}\right)}{\partial e_{i j}} e_{i j}+\frac{\partial F\left(0,0, T_{0}\right)}{\partial \kappa_{i j}} \kappa_{i j}+\frac{\partial F\left(0,0, T_{0}\right)}{\partial T}\left(T-T_{0}\right) \\
& +\frac{1}{2} \frac{\partial^{2} F\left(0,0, T_{0}\right)}{\partial e_{i j} \partial e_{k l}} e_{i j} e_{k l}+\frac{1}{2} \frac{\partial^{2} F\left(0,0, T_{0}\right)}{\partial \kappa_{i j} \partial \kappa_{k l}} \kappa_{i j} \kappa_{k l}+\frac{\partial F\left(0,0, T_{0}\right)}{\partial e_{i j} \partial \kappa_{k l}} e_{i j} \kappa_{k l} \\
& +\frac{\partial^{2} F\left(0,0, T_{0}\right)}{\partial e_{i j} \partial T} e_{i j}\left(T-T_{0}\right)+\frac{\partial^{2} F\left(0,0, T_{0}\right)}{\partial \kappa_{i j} \partial T} \kappa_{i j}\left(T-T_{0}\right)+\frac{1}{2} \frac{\partial^{2} F\left(0,0, T_{0}\right)}{\partial T^{2}}\left(T-T_{0}\right)^{2}+\cdots
\end{aligned}
$$

The constant $F\left(0,0, T_{0}\right)$ is the free energy corresponding to the reference state, which can be taken arbitrarily equal to zero. Since there is no initial stresses in the reference state, the quantities $\frac{\partial F\left(0,0, T_{0}\right)}{\partial e_{i j}}$ and $\frac{\partial F\left(0,0, T_{0}\right)}{\partial \kappa_{i j}}$ also vanish. By assuming the entropy to be zero at the reference state, we take $\frac{\partial F\left(0,0, T_{0}\right)}{\partial T}$ equal zero. Therefore, for linear elastic sizedependent thermoelasicity the free energy $F$ can be considered as the homogeneous quadratic

$$
F=\frac{1}{2} A_{i j k l} e_{i j} e_{k l}+\frac{1}{2} B_{i j k l} \kappa_{i j} \kappa_{k l}+C_{i j k l} e_{i j} \kappa_{k l}-a_{i j} e_{i j} \vartheta-b_{i j} \kappa_{i j} \vartheta-\frac{1}{2} m \vartheta^{2}
$$

where the coefficients are defined as

$$
A_{i j k l}=\frac{\partial^{2} F\left(0,0, T_{0}\right)}{\partial e_{i j} \partial e_{k l}}, B_{i j k l}=\frac{\partial^{2} F\left(0,0, T_{0}\right)}{\partial \kappa_{i j} \partial \kappa_{k l}}, C_{i j k l}=\frac{\partial F\left(0,0, T_{0}\right)}{\partial e_{i j} \partial \kappa_{k l}}
$$




$$
\begin{gathered}
a_{i j}=-\frac{\partial^{2} F\left(0,0, T_{0}\right)}{\partial e_{i j} \partial T}, b_{i j}=-\frac{\partial^{2} F\left(0,0, T_{0}\right)}{\partial \kappa_{i j} \partial T} \\
m=-\frac{\partial^{2} F\left(0,0, T_{0}\right)}{\partial T^{2}}
\end{gathered}
$$

Obviously, these tensors have the symmetry and skew-symmetry relations

$$
\begin{gathered}
A_{i j k l}=A_{k l i j}=A_{j i k l} \\
B_{i j k l}=B_{k l i j}=-B_{j i k l} \\
C_{i j k l}=C_{j i k l}=-C_{i j l k} \\
a_{i j}=a_{j i j} \\
b_{i j}=-b_{j i}
\end{gathered}
$$

Since the material is heterogeneous, the mechanical and thermal tensor properties can vary from point to point in space. However, we have assumed that these properties are constants at each point during the deformation and temperature change.

Consequently, by using the Helmholtz free energy density Eq. (68) in the general relations (35-37), we obtain the following constitutive relations

$$
\begin{gathered}
\sigma_{(j i)}=A_{i j k l} e_{k l}+C_{i j k l} \kappa_{k l}-a_{i j} \vartheta \\
\mu_{j i}=B_{i j k l} \kappa_{k l}+C_{k l i j} e_{k l}-b_{i j} \vartheta \\
S=a_{i j} e_{i j}+b_{i j} \kappa_{i j}+m \vartheta
\end{gathered}
$$

The tensors $a_{i j}$ and $b_{i j}$ represent the thermoelastic properties of the material. The symmetric true tensor $a_{i j}$ is the coupling term between the temperature change $\vartheta$ and the symmetric part of force-stress tensor $\sigma_{(j i)}$ in Eq. (77), and the skew-symmetric pseudo tensor $b_{i j}$ is the size-dependent coupling term between the temperature and the couple-stress tensor $\mu_{j i}$ in Eq. (78). We will shortly explore further characters of these thermoelastic tensors and the 
quantity $m$. Consequently, for the most general case, the number of distinct components for $A_{i j k l}, B_{i j k l}, C_{i j k l}, a_{i j}, b_{i j}$ and are 21,6,18,6, and 3, respectively. Therefore, the most general linear thermoelastic anisotropic material, including $m$, is described by 55 independent constitutive coefficients.

The equations (77) and (78) can be considered as the generalized Duhamel-Neumann relations for a heterogeneous anisotropic size-dependent body. Interestingly, by using entropy density Eq. (79) and free energy Eq. (68) in Eq. (28), we obtain

$$
U=F+S T=\frac{1}{2} A_{i j k l} e_{i j} e_{k l}+\frac{1}{2} B_{i j k l} \kappa_{i j} \kappa_{k l}+C_{i j k l} e_{i j} \kappa_{k l}+\frac{1}{2} m \vartheta^{2}
$$

for density function $U$. This expression is a positive definite quadratic form without explicit thermomechanical coupling. This shows that the tensors $A_{i j k l}, B_{i j k l}$ and $C_{i j k l}$ containing the elastic constitutive coefficients, and the parameter $m$ are positive definite. The tensor $A_{i j k l}$ is actually equivalent to its corresponding tensor in Cauchy elasticity.

It is interesting to note that the Helmholtz free energy density $F$ can also be written in terms of the mean curvature vector as

$$
F=\frac{1}{2} A_{i j k l} e_{i j} e_{k l}+\frac{1}{2} B_{i j} \kappa_{i} \kappa_{j}+C_{i j k} e_{i j} \kappa_{k}-a_{i j} e_{i j} \vartheta-b_{i} \kappa_{i} \vartheta-\frac{1}{2} m \vartheta^{2}
$$

where

$$
\begin{gathered}
B_{i j k l}=\frac{1}{4} \varepsilon_{i j p} \varepsilon_{k l q} B_{p q} \\
C_{i j k l}=\frac{1}{2} C_{i j m} \varepsilon_{m l k} \\
b_{i j}=\frac{1}{2} \varepsilon_{m j i} b_{m}
\end{gathered}
$$

and the symmetry relations

$$
B_{i j}=B_{j i}
$$




$$
C_{i j k}=C_{j i k}
$$

hold. It is seen that the tensor $B_{i j}$ is positive definite. Note that the number of distinct components for true tensors $B_{i j}$ and $C_{i j k}$, and true vector $b_{i}$ are 6,18 and 3 , respectively. By using the Helmholtz free energy density Eq. (81) in the general relations (35), (46) and (37), we obtain the following constitutive relations

$$
\begin{gathered}
\sigma_{(j i)}=A_{i j k l} e_{k l}+C_{i j k} \kappa_{k}-a_{i j} \vartheta \\
\mu_{i}=-\frac{1}{2} B_{i j} \kappa_{j}-\frac{1}{2} C_{k j i} e_{k j}+\frac{1}{2} b_{i} \vartheta \\
S=a_{i j} e_{i j}+b_{i} \kappa_{i}+m \vartheta
\end{gathered}
$$

The skew-symmetric part of the force-stress tensor for a homogeneous material is found as

$$
\sigma_{[j i]}=-\mu_{[i, j]}=\frac{1}{4} B_{i m} \kappa_{m, j}-\frac{1}{4} B_{j m} \kappa_{m, i}+\frac{1}{4} C_{k m i} e_{k m, j}-\frac{1}{4} C_{k m j} e_{k m, i}-\frac{1}{4} b_{i} \vartheta_{, j}+\frac{1}{4} b_{j} \vartheta_{, i}
$$

We notice the appearance of the third order gradient of deformation $\kappa_{i, j}$ in Eq. (90) for the skew-symmetric part of force-stress tensor $\sigma_{[j i]}$ is the result of angular equation of motion (18), which is not a constitutive equation. Consequently, the constitutive relation for the total force-stress tensor in a homogeneous material is

$$
\begin{aligned}
& \sigma_{j i}=A_{i j k l} e_{k l}+C_{i j k} \kappa_{k}-a_{i j} \vartheta+\frac{1}{4} B_{i m} \kappa_{m, j}-\frac{1}{4} B_{j m} \kappa_{m, i} \\
& +\frac{1}{4} C_{k m i} e_{k m, j}-\frac{1}{4} C_{k m j} e_{k m, i}-\frac{1}{4} b_{i} \vartheta_{, j}+\frac{1}{4} b_{j} \vartheta_{, i}
\end{aligned}
$$

The physical character of the symmetric tensor $a_{i j}$ and skew-symmetric tensor $b_{i j}$ can be investigated by writing Eqs. (77) and (78) as

$$
\begin{gathered}
\sigma_{(j i)}=A_{i j k l}\left(e_{k l}-\alpha_{k l} \vartheta\right)+C_{i j k l}\left(\kappa_{k l}-\beta_{k l} \vartheta\right) \\
\mu_{j i}=B_{i j k l}\left(\kappa_{k l}-\beta_{k l} \vartheta\right)+C_{k l i j}\left(e_{k l}-\alpha_{k l} \vartheta\right)
\end{gathered}
$$

where 


$$
\begin{aligned}
& A_{i j k l} \alpha_{k l}+C_{i j k l} \beta_{k l}=a_{i j} \\
& C_{k l i j} \alpha_{k l}+B_{i j k l} \beta_{k l}=b_{i j}
\end{aligned}
$$

The true tensor $\alpha_{i j}$ and pseudo tensor $\beta_{i j}$ are the new thermoelastic tensors with the obvious symmetry and skew-symmetry relations

$$
\begin{aligned}
& \alpha_{i j}=\alpha_{j i} \\
& \beta_{i j}=-\beta_{j i}
\end{aligned}
$$

It is seen that the quantities $\alpha_{i j} \vartheta$ and $\beta_{i j} \vartheta$ in Eqs. (92) and (93) are the strain and mean curvature tensors created by the temperature change $\vartheta=T-T_{0}$, respectively. Hence, they represent the thermal strain and thermal mean curvature tensors in an unrestrained body

$$
\begin{aligned}
& e_{i j}^{T h}=\alpha_{i j} \vartheta \\
& \kappa_{i j}^{T h}=\beta_{i j} \vartheta
\end{aligned}
$$

While the symmetric tensor $\alpha_{i j}$ is the classical coefficient of thermal expansion tensor, the new size-dependent skew-symmetric tensor $\beta_{i j}$ may be called the coefficient of thermal flexion tensor. Although, there is generally no restriction on the tensors $\alpha_{i j}$ and $\beta_{i j}$, in most cases $\alpha_{i j}$ is positive definite. It is obvious that for the most general anisotropic case, the number of distinct components for $\alpha_{i j}$ and $\beta_{i j}$ are 6 , and 3 , respectively. Consequently, the constitutive relations (92) and (93) can be written as

$$
\begin{gathered}
\sigma_{(j i)}=A_{i j k l}\left(e_{k l}-e_{k l}^{T h}\right)+C_{i j k l}\left(\kappa_{k l}-\kappa_{k l}^{T h}\right) \\
\mu_{j i}=B_{i j k l}\left(\kappa_{k l}-\kappa_{k l}^{T h}\right)+C_{k l i j}\left(e_{k l}-e_{k l}^{T h}\right)
\end{gathered}
$$

The tensors $e_{i j}-e_{i j}^{T h}$ and $\kappa_{i j}-\kappa_{i j}^{T h}$ represent the mechanical strain and mean curvature tensors, respectively. Similarly, Eqs. (87) and (88) can also be written as 


$$
\begin{gathered}
\sigma_{(j i)}=A_{i j k l}\left(e_{k l}-\alpha_{k l} \vartheta\right)+C_{i j k}\left(\kappa_{k}-\beta_{k} \vartheta\right) \\
\mu_{i}=-\frac{1}{2} B_{i j}\left(\kappa_{j}-\beta_{j} \vartheta\right)-\frac{1}{2} C_{k j i}\left(e_{k j}-\alpha_{k j} \vartheta\right)
\end{gathered}
$$

where

$$
\begin{gathered}
A_{i j k l} \alpha_{k l}+C_{i j k} \beta_{k}=a_{i j} \\
C_{k j i} \alpha_{k j}+B_{i j} \beta_{j}=b_{i}
\end{gathered}
$$

These set of 9 independent equations (104) and (105) give 9 independent coefficients $\alpha_{i j}$ and $\beta_{i}$. The dual relation between thermal flexion true vector $\beta_{i}$ and thermal flexion pseudo tensor $\beta_{i j}$ is given by

$$
\beta_{i}=\varepsilon_{i j k} \beta_{k j}
$$

Hence, the thermal mean curvature vector in the unrestrained body is defined as

$$
\kappa_{i}^{T h}=\beta_{i} \vartheta
$$

Therefore

$$
\begin{gathered}
\sigma_{(j i)}=A_{i j k l}\left(e_{k l}-e_{k l}^{T h}\right)+C_{i j k}\left(\kappa_{k}-\kappa_{k}^{T h}\right) \\
\mu_{i}=-\frac{1}{2} B_{i j}\left(\kappa_{j}-\kappa_{j}^{T h}\right)-\frac{1}{2} C_{k j i}\left(e_{k j}-e_{k j}^{T h}\right)
\end{gathered}
$$

It should be noticed that the effect of thermal flexion moduli always appears along with couple-stresses. This means that if we neglect the couple stresses $\left(B_{i j}=0, C_{i j k}=0\right)$, all other size-dependent effects disappear as well $\left(\beta_{i j}=0\right)$.

Now, we try to define the positive quantity $m$ in the Helmholtz free energy Eq. (81). For this purpose, let us return to the Taylor expansion Eq. (67) for Helmholtz free energy and neglect all powers higher than second in $e_{i j}$ and $\kappa_{i j}$, but keep all terms depending on tem- 
perature and collect them in one function $C=C(\vartheta)$. Consequently, for Helmholtz free energy we have

$$
F \doteq \frac{1}{2} A_{i j k l} e_{i j} e_{k l}+\frac{1}{2} B_{i j k l} \kappa_{i j} \kappa_{k l}+C_{i j k l} e_{i j} \kappa_{k l}-a_{i j} e_{i j} \vartheta-b_{i j} \kappa_{i j} \vartheta+C(\vartheta)
$$

where $C=C(\vartheta)$ is a function depending on temperature term only with $C(0)=0$. By using this form of $F$ in the entropy density function Eq. (37), we obtain

$$
S=a_{i j} e_{i j}+b_{i} \kappa_{i}-\frac{\partial C(\vartheta)}{\partial T}
$$

Consequently, Eq. (52) gives

$$
c_{v}=T\left(\frac{\partial S}{\partial T}\right)_{e_{i j,}, x_{i j}}=-T \frac{\partial^{2} C(\vartheta)}{\partial T^{2}}
$$

which can be written as

$$
\frac{\partial^{2} C(\vartheta)}{\partial T^{2}}=-\frac{c_{v}}{T}
$$

By integrating this relation, and noticing that at the natural state, $F=0$ and $S=0$, we obtain

$$
\frac{\partial C(\vartheta)}{\partial T}=-\int_{T_{0}}^{T} \frac{c_{v}}{T} d T
$$

For the linear theory $c_{v}$ is constant. Thus, this integral becomes

$$
-\frac{\partial C(\vartheta)}{\partial T}=c_{v} \ln \frac{T}{T_{0}}
$$


By using this in Eq. (111) for the entropy density, we obtain

$$
S=a_{i j} e_{i j}+b_{i} \kappa_{i}+c_{v} \ln \frac{T}{T_{0}}=a_{i j} e_{i j}+b_{i} \kappa_{i}+c_{v} \ln \left(1+\frac{\vartheta}{T_{0}}\right)
$$

However, by the restriction $\left|\frac{\vartheta}{T_{0}}\right|=\left|\frac{T-T_{0}}{T_{0}}\right|<<1$, for small temperature change, this relation can be written as

$$
S=a_{i j} e_{i j}+b_{i} \kappa_{i}+\frac{c_{v}}{T_{0}} \vartheta
$$

By comparing Eqs. (117) and (89), we find

$$
m=\frac{c_{v}}{T_{0}}
$$

Therefore, when $\left|\frac{\vartheta}{T_{0}}\right| \ll<1$

$$
F=\frac{1}{2} A_{i j k l} e_{i j} e_{k l}+\frac{1}{2} B_{i j} \kappa_{i} \kappa_{j}+C_{i j k} e_{i j} \kappa_{k}-a_{i j} e_{i j} \vartheta-b_{i} \kappa_{i} \vartheta-\frac{c_{v}}{2 T_{0}} \vartheta^{2}
$$

and

$$
U=F+S T=\frac{1}{2} A_{i j k l} e_{i j} e_{k l}+\frac{1}{2} B_{i j} \kappa_{i} \kappa_{j}+C_{i j k} e_{i j} \kappa_{k}+\frac{c_{v}}{2 T_{0}} \vartheta^{2}
$$

When the constitutive relations force-stress tensor (91), entropy density (117) and thermal flux vector (63) are written in terms of displacements and temperature for homogeneous material, we obtain

$$
\begin{aligned}
& \sigma_{j i}=A_{i j k l} u_{k, l}+\frac{1}{4} C_{i j k}\left(u_{m, m k}-\nabla^{2} u_{k}\right)+\frac{1}{4} C_{k m i} u_{k, m j}-\frac{1}{4} C_{k m j} u_{k, m i} \\
& +\frac{1}{16} B_{i k}\left(u_{m, m l j}-\nabla^{2} u_{k, j}\right)-\frac{1}{16} B_{j k}\left(u_{m, m k i}-\nabla^{2} u_{k, i}\right)-a_{i j} \vartheta-\frac{1}{4} b_{i} \vartheta_{, j}+\frac{1}{4} b_{j} \vartheta_{, i}
\end{aligned}
$$




$$
\begin{gathered}
S=a_{i j} u_{i, j}+\frac{1}{4} b_{i}\left(u_{m, m i}-\nabla^{2} u_{i}\right)+\frac{c_{v}}{T_{0}} \vartheta \\
q_{i}=-k_{i j} T_{, j}
\end{gathered}
$$

By carrying these forms into the linear motion Eq. (16) and the entropy balance Eq. (41), one obtains the governing equations for size-dependent homogeneous thermoelasticity as follows:

$$
\begin{gathered}
A_{i j k l} u_{k, l j}+\frac{1}{4} C_{i j k}\left(u_{m, m j k}-\nabla^{2} u_{k, j}\right)+\frac{1}{4} C_{k m i} \nabla^{2} u_{k, m}-\frac{1}{4} C_{k m j} u_{k, m i j} \\
+\frac{1}{16} B_{i k}\left(\nabla^{2} u_{m, m k}-\nabla^{2} \nabla^{2} u_{k}\right)-\frac{1}{16} B_{j k}\left(u_{m, m k i j}-\nabla^{2} u_{k, i j}\right) \\
-a_{i j} T_{, j}-\frac{1}{4} b_{i} T_{, j j}+\frac{1}{4} b_{j} T_{, i j}+\rho f_{i}=\rho \ddot{u}_{i} \\
\left(a_{i j} \dot{e}_{i j}+b_{i} \dot{\kappa}_{i}\right) T_{0}+c_{v} \dot{T}=k_{i j} T_{, i j}+Q
\end{gathered}
$$

These are the coupled size-dependent thermoelasticity equations. It should be noticed that the expression $\left(a_{i j} \dot{e}_{i j}+b_{i} \dot{K}_{i}\right) T_{0}$ is the coupling term in the entropy balance Eq. (125), which involves the thermal and mechanical terms. If this term can be neglected, we obtain the uncoupled heat conduction equation

$$
c_{v} \dot{T}=k_{i j} T_{, i j}+Q
$$

which is no longer size-dependent.

As we mentioned, the prescribed boundary conditions on the surface of the body can be any compatible combination of $u_{i}, \omega_{i}, T$ and $t_{i}^{(n)}, m_{i}^{(n)}, q^{(n)}$. The force-traction vector $t_{i}^{(n)}$, moment-traction $m_{i}^{(n)}$ and normal heat flux $q^{(n)}$ at any point on a surface $A$, with outward unit normal vector $n_{i}$, are 


$$
\begin{gathered}
t_{i}^{(n)}=\sigma_{j i} n_{j} \\
=\left(\begin{array}{c}
A_{i j k l} e_{k l}+C_{i j k} \kappa_{k}-a_{i j} \vartheta+\frac{1}{4} B_{i m} \kappa_{m, j}-\frac{1}{4} B_{j m} \kappa_{m, i} \\
+\frac{1}{4} C_{k m i} e_{k m, j}-\frac{1}{4} C_{k m j} e_{k m, i}-\frac{1}{4} b_{i} \vartheta_{, j}+\frac{1}{4} b_{j} \vartheta_{, i}
\end{array}\right) n_{j} \\
m_{i}^{(n)}=\varepsilon_{i j k} \mu_{k} n_{j}=\varepsilon_{i j k}\left(-\frac{1}{2} B_{k m} \kappa_{m}-\frac{1}{2} C_{m n k} e_{m n}+\frac{1}{2} b_{k} \vartheta\right) n_{j} \\
q^{(n)}=q_{i} n_{i}=-k_{i j} T_{, j} n_{i}
\end{gathered}
$$

\section{ISOTROPIC LINEAR THERMOELASTIC MATERIAL}

For a heterogeneous isotropic material, the symmetry relations require

$$
\begin{gathered}
k_{i j}=k \delta_{i j} \\
A_{i j k l}=\lambda \delta_{i j} \delta_{k l}+\mu \delta_{i k} \delta_{j l}+\mu \delta_{i l} \delta_{j k} \\
B_{i j}=16 \eta \delta_{i j} \\
C_{i j k}=0 \\
\alpha_{i j}=\alpha \delta_{i j} \\
\beta_{i j}=0, \quad \beta_{i}=0 \\
a_{i j}=a \delta_{i j} \\
b_{i j}=0, \quad b_{i}=0
\end{gathered}
$$

where the quantities can vary in the domain from point to point. The moduli $\lambda$ and $\mu$ have the same meaning as the Lamé constants for an isotropic material in Cauchy elasticity. As we know, these two constants are related by 


$$
\lambda=2 \mu \frac{v}{1-2 v}=\frac{v E}{(1+v)(1-2 v)}
$$

where $v$ is the Poisson ratio, and $E$ is the modulus of elasticity or Young's modulus. It is seen that

$$
3 \lambda+2 \mu=2 \mu \frac{1+v}{1-2 v}=\frac{E}{1-2 v}=3 K
$$

where $K$ is the bulk modulus of elasticity $K=\frac{E}{3(1-2 v)}$. We also notice that the material constant $\eta$ accounts for the couple-stresses in the isotropic material.

It is seen that for the isotropic material the coefficient of thermal flexion tensor $\beta_{i j}$ vanishes and there is only one coefficient of the thermal expansion $\alpha$. Therefore, the relations (98) and (99) show

$$
\begin{gathered}
e_{i j}^{T h}=\alpha \delta_{i j} \vartheta \\
\kappa_{i}^{T h}=0
\end{gathered}
$$

This means that no thermal shear strain and thermal mean curvature are created by the temperature change $\vartheta$. As a result, for the tensor $a_{i j}$, we obtain

$$
a_{i j}=A_{i j k l} \alpha_{k l}=(3 \lambda+2 \mu) \alpha \delta_{i j}
$$

which shows that

$$
a=(3 \lambda+2 \mu) \alpha=3 K \alpha
$$

As a result, the Helmholtz free energy and internal energy densities become

$$
F=\frac{1}{2} \lambda e_{j j} e_{k k}+\mu e_{i j} e_{i j}+8 \eta \kappa_{i} \kappa_{i}-\frac{1}{2} \frac{c_{v}}{T_{0}} \vartheta^{2}-(3 \lambda+2 \mu) \alpha \vartheta e_{k k}
$$




$$
S=\frac{c_{v}}{T_{0}} \vartheta+(3 \lambda+2 \mu) \alpha e_{k k}
$$

respectively. Therefore

$$
U=\frac{1}{2} \lambda e_{j j} e_{k k}+\mu e_{i j} e_{i j}+8 \eta \kappa_{i} \kappa_{i}+\frac{c_{v}}{T_{0}} \vartheta^{2}
$$

The following restrictions are necessary for positive definite energy density $U$

$$
3 \lambda+2 \mu>0, \quad \mu>0, \quad \eta>0, \quad c_{v}>0
$$

The ratio

$$
\frac{\eta}{\mu}=l^{2}
$$

specifies the characteristic material length $l$, which accounts for size-dependency in the small deformation couple stress elasticity theory under consideration here.

Consequently, the constitutive relations for the symmetric part of the force-stress tensor, the couple-stress vector, and the heat flux vector can be written as

$$
\begin{gathered}
\sigma_{(j i)}=\lambda e_{k k} \delta_{i j}+2 \mu e_{i j}-(3 \lambda+2 \mu) \alpha \vartheta \\
\mu_{i}=-8 \mu l^{2} \kappa_{i} \\
q_{i}=-k \vartheta_{, i}
\end{gathered}
$$

Interestingly, Eq. (149) can be written as

$$
e_{i j}-e_{i j}^{T h}=\frac{1}{E}\left[(1+v) \sigma_{(j i)}-v \sigma_{(k k)} \delta_{i j}\right]
$$

or

$$
e_{i j}=\frac{1}{E}\left[(1+v) \sigma_{(j i)}-v \sigma_{(k k)} \delta_{i j}\right]+\alpha\left(T-T_{0}\right) \delta_{i j}
$$


For the skew-symmetric part of the force-stress tensor in a homogeneous material, we have

$$
\sigma_{[j i]}=-\mu_{[i, j]}=2 \mu l^{2} \nabla^{2} \omega_{j i}
$$

which can also be written as

$$
\sigma_{[j i]}=2 \mu l^{2} \varepsilon_{i j k} \nabla^{2} \omega_{k}
$$

Therefore, for the total force-stress tensor, we have

$$
\sigma_{j i}=\lambda e_{k k} \delta_{i j}+2 \mu e_{i j}+2 \mu l^{2} \nabla^{2} \omega_{j i}-(3 \lambda+2 \mu) \alpha\left(T-T_{0}\right)
$$

For the governing equations, we have

$$
\begin{gathered}
{\left[\lambda+\mu\left(1+l^{2} \nabla^{2}\right)\right] u_{k, k i}+\mu\left(1-l^{2} \nabla^{2}\right) \nabla^{2} u_{i}-(3 \lambda+2 \mu) \alpha T_{, i}+\rho f_{i}=\rho \ddot{u}_{i}} \\
(3 \lambda+2 \mu) \alpha T_{0} \dot{e}_{k k}+c \dot{T}=k \nabla^{2} T+Q
\end{gathered}
$$

These are the coupled size-dependent thermoelasticity equations for a homogeneous isotropic material. As we noticed, the expression $(3 \lambda+2 \mu) \alpha T_{0} \dot{e}_{k k}$ is the coupling term in the energy Eq. (158), which involves the thermal and mechanical terms. If this term can be neglected, we obtain the uncoupled conduction equation

$$
c_{v} \dot{T}=k_{i j} T_{i j}+Q
$$

The governing equations (157) and (158) can also be written in vectorial form as

$$
\begin{gathered}
{\left[\lambda+\mu\left(1+l^{2} \nabla^{2}\right)\right] \nabla(\nabla \bullet \mathbf{u})+\mu\left(1-l^{2} \nabla^{2}\right) \nabla^{2} \mathbf{u}-(3 \lambda+2 \mu) \alpha \nabla T+\rho \mathbf{f}=\rho \ddot{\mathbf{u}}} \\
(3 \lambda+2 \mu) \alpha T_{0} \nabla \bullet \dot{\mathbf{u}}+c_{v} \dot{T}=k \nabla^{2} T+Q
\end{gathered}
$$

Based on the Helmholtz decomposition theorem, we can introduce the scalar potential $\phi$ and vector potential A such that

$$
\mathbf{u}=\nabla \phi+\nabla \times \mathbf{A}
$$


where the first and second terms in the right hand side of Eq. (162) represent the dilatational (irrotational) and equivoluminal (shear) components of deformation, respectively.

Because of gauge freedom, we can arbitrarily take the gauge condition

$$
\nabla \bullet \mathbf{A}=0
$$

As a result, the equation of motion (160) can be decomposed in two equations for the potentials $\phi$ and $\mathbf{A}$ as

$$
\begin{gathered}
(\lambda+2 \mu) \nabla^{2} \phi-(3 \lambda+2 \mu) \alpha T+\rho f_{\phi}=\rho \ddot{\phi} \\
\mu\left(1-l^{2} \nabla^{2}\right) \nabla^{2} \mathbf{A}+\rho \mathbf{f}_{\mathbf{A}}=\rho \ddot{\mathbf{A}}
\end{gathered}
$$

where we have assumed the decomposition

$$
\mathbf{f}=\nabla f_{\phi}+\nabla \times \mathbf{f}_{\mathbf{A}}
$$

When the heat sources and body forces are absent, we obtain the governing equations

$$
\begin{gathered}
c_{1}^{2} \nabla^{2} \phi-(3 \lambda+2 \mu) \frac{1}{\rho} \alpha T=\ddot{\phi} \\
c_{2}^{2}\left(1-l^{2} \nabla^{2}\right) \nabla^{2} \mathbf{A}=\ddot{\mathbf{A}} \\
(3 \lambda+2 \mu) \alpha T_{0} \nabla^{2} \dot{\phi}+c_{v} \dot{T}=k \nabla^{2} T
\end{gathered}
$$

where $c_{1}$ and $c_{2}$ are the velocities of dilatational and shear waves in classical elastic waves, respectively, defined as

$$
c_{1}^{2}=\frac{\lambda+2 \mu}{\rho}, \quad c_{2}^{2}=\frac{\mu}{\rho}
$$

It is seen that the dilatational (irrotational) wave equation (167) is coupled to the temperature equation (169). This means that the propagation of dilatational wave is accompanied by a generation of heat. Therefore, the dilatational wave is damped and its energy is partly converted to heat. However, the shear wave equation (168) is uncoupled from the tempera- 
ture. This means that this wave is not damped, but undergoes dispersion, as a result of sizedependency. Furthermore, the shear wave does not generate any temperature change.

\section{CONCLUSIONS}

The consistent size-dependent continuum mechanics is a practical theory, which enables us to develop many different multi-physics formulations that may govern the behavior of solid continua at the smallest scales. The size-dependent formulations have the priority because of their importance in nanomechanics and nanotechnology. Here, we have developed the sizedependent thermoelascticity, which shows the possible thermal mean curvature in anisotropic material. The most general anisotropic linear elastic material is described by 55 independent constitutive coefficients. This includes three thermal flexion coefficients relating thermal mean curvatures to temperature change.

The new size dependent thermoelascticity clearly shows that the thermal mean curvature disappears in isotropic couple stress materials, where the two Lamé parameters, one length scale, and one thermal expansion completely characterize the behavior.

The present theory shows that couple-stresses are necessary for the development of any thermomechanical size-dependent effect. Additional aspects of linear thermoelasticity, including reciprocal theorem, fundamental solutions and computational mechanics formulations, will be addressed in forthcoming work. Beyond this, the present theory should be useful for the development of other coupled size-dependent multi-physics formulations, such as piezothermo-elasticity, which are also important for analysis at small scales.

\section{References}

Ahmadi, G., Firoozbakhsh, K., (1975). First strain gradient theory of thermoelasticity. International Journal of Solids and Structures, 11: 339-345.

Biot, M. A., (1956). Thermoelasticity and irreversible thermodynamics. Journal of Applied Physics, 27: 240-253.

Carslaw, H. S., Jaeger, J. C., (1959). Conduction of Heat in Solids. Clarendon Press, Oxford.

Duhamel, J.-M.-C., (1832). Sur les Équations Générales de la Propagation de la Chaleur dans les Corps Solides dont la Conductibilité n'est pas la Même dans Tous les Sens. J. Ec. Polytech. Paris, 13(21): 356399.

Eringen, A. C., (1970). Foundations of micropolar thermoelasticity. International Center for Mechanical Science, Courses and Lectures, no.23, Springer, Berlin.

Hadjesfandiari, A. R., Dargush, G. F., (2011). Couple stress theory for solids. International Journal of Solids and Structures, 48(18): 2496-2510.

Hadjesfandiari, A. R., (2013). Size-dependent piezoelectricity. International Journal of Solids and Structures, 50 (18): 2781-2791.

Koiter, W. T., (1964). Couple stresses in the theory of elasticity, I and II. Proceedings of the Koninklijke Nederlandse Akademie van Wetenschappen, Series B, 67: 17-44.

Lord, H. W, Shulman, Y., (1967). A generalized dynamical theory of thermoelasticity. Journal of the Mechanics and Physics of Solids, 15: 299-309. 
Mindlin, R. D., (1964). Micro-structure in linear elasticity. Archive for Rational Mechanics and Analysis, 16: $51-78$.

Mindlin, R. D., Eshel N. N., (1968). On first strain-gradient theories in linear elasticity. International Journal of Solids and Structures, 4: 109-124.

Mindlin, R. D., Tiersten, H. F., (1962). Effects of couple-stresses in linear elasticity. Archive for Rational Mechanics and Analysis, 11: 415-488.

Nowacki, W., (1962). Thermoelasticity. Addison-Wesley, Reading, Massachusetts.

Nowacki, W., (1966a). Couple stresses in the theory of thermoelasticity i. Bulletin of Polish Academy of Sciences Series, Science and Technology, 14: 129-138.

Nowacki, W., (1966b). Couple stresses in the theory of thermoelasticity ii. Bulletin of Polish Academy of Sciences Series, Science and Technology, 14: 263-272.

Nowacki, W., (1966c). Couple stresses in the theory of thermoelasticity iii. Bulletin of Polish Academy of Sciences Series, Science and Technology, 14: 801-809.

Onsager, L., (1931). Reciprocal relations in irreversible processes. I. Physical Review Letters, 37: 405-426. 